\title{
Neuralgia del Trigémino, del Glosofaríngeo y Post- herpética en Pacientes del Hospital Universitario San Ignacio (HUSI) Bogota, Colombia
}

\author{
Trigeminal, Glossopharyngeal and Post-Herpetic Neuralgia in Patients \\ of the San Ignacio University Hospital (HUSI) Bogota, Colombia
}

\begin{abstract}
León-Corredor Maryorie'; Fonseca-Rubio María Angelica ${ }^{2}$; Velosa-Porras Juliana; Rodríguez-Ciodaro Adriana ${ }^{3}$ \& Barrientos-Sánchez Silvia ${ }^{3}$
\end{abstract}

\begin{abstract}
LEÓN-CORREDOR, M.; FONSECA-RUBIO, M. A.; VELOSA-PORRAS, J.; RODRÍGUEZ-CIODARO, A. \& BARRIENTOSSÁNCHEZ, S. Neuralgia del trigémino, del glosofaríngeo y postherpética en pacientes del Hospital Universitario San Ignacio (HUSI) Bogota, Colombia. Int. J. Odontostomat., 15(3):688-693, 2021.

RESUMEN: Determinar las características clínicas, pautas para el diagnóstico, tratamiento y evolución de la neuralgia del trigémino, postherpética y del nervio glosofaríngeo. Se realizó un estudio descriptivo de 119 historias clínicas de pacientes diagnosticados con neuralgia del trigémino, glosofaríngeo y postherpética tratados en el Hospital Universitario San Ignacio, registrando datos de sexo, edad, antecedentes médicos, exámenes complementarios, características clínicas, tratamientos y evolución. Se revisaron 119 historias clínicas, de las cuales 86 pertenecían a mujeres y 33 a hombres, el $84 \%$ correspondían a neuralgias del trigémino, el $11,7 \%$ a post- herpética, y el 2,5\% en el glosofaríngeo. El lado afectado más común fue el lado izquierdo con un $54 \%$, las ramas más afectadas en la neuralgia del trigémino fue la maxilar y mandibular (V2V3), en la postherpética la oftálmica (V1), la patología concomitante más frecuente hallada en el grupo fue la HTA, el tipo de dolor más frecuente fue el lancinante con un $82,4 \%$. Los medicamentos más utilizados fueron la carbamazepina (60,5\%) seguida de pregabalina $(29,4 \%)$, en tratamientos quirúrgicos la radiofrecuencia fue la más empleada en un $32 \%$. 30 pacientes presentaron recaídas que en general fueron manejadas con aumento de las dosis de los medicamentos. Este estudio muestra una similitud con la literatura reportada, presentando más casos de neuralgia de trigémino, seguida de la post herpética y por último del glosofaríngeo. Más frecuente en mujeres, con un promedio de edad de 60 años, afectando en mayor proporción la rama mandibular y maxilar en la neuralgia de trigémino y en la postherpética la oftálmica.
\end{abstract}

PALABRAS CLAVE: neuralgia trigémino, neuralgia postherpética, neuralgia glosofaríngeo, tratamiento de neuralgias.

\section{INTRODUCCIÓN}

En odontología los síndromes dolorosos faciales son variados y de distinta etiología y pueden generar confusión al momento del diagnóstico llevando a exodoncias o endodoncias que no solucionan el problema. Las neuralgias se describen como una rara forma de dolor neuropático que aparece repentinamente e incluyen a la neuralgia del trigémino (NT) una de las más comunes, suele ser unilateral asociada a dolor breve, punzante, presentándose más del lado derecho, con un dolor recurrente en cualquiera de sus tres ramas (Cruccu et al., 2016) (Sathasivam et al., 2017). La neuralgia postherpética(NPH) asociada a la infección previa por Herpes Zoster se presenta con dolor lancinante persistente en el trayecto nervioso trigeminal (Kawai et al., 2015) y la neuralgia del glosofaríngeo (NGF) es un trastorno doloroso unilateral, que semeja a descargas eléctricas en el oído, la base de la lengua, la fosa tonsilar o debajo del ángulo de la mandíbula. (Teixeira et al., 2008) (Singh et al., 2013) En general, el diagnóstico se fundamenta en

\footnotetext{
${ }^{1}$ Programa de práctica clínica, State Universityof Medicine and Pharmacy (USMF), NicolaeTestemitanu. Republic of Moldova.

${ }^{2}$ Práctica privada en Patología Bucal.

${ }^{3}$ Centro Investigaciones Odontológicas, Facultad de Odontología, Pontificia Universidad Javeriana. Bogotá, Colombia.
} 
LEÓN-CORREDOR, M.; FONSECA-RUBIO, M. A.; VELOSA-PORRAS, J.; RODRÍGUEZ-CIODARO, A. \& BARRIENTOS-SÁNCHEZ, S. Neuralgia del trigémino, del glosofaríngeo y postherpética en pacientes del Hospital Universitario San Ignacio (HUSI) Bogota, Colombia. Int. J. Odontostomat., 15(3):688-693, 2021.

los hallazgos clínicos, aunque se suelen usar ayudas como la resonancia magnética y la tomografía que permiten identificar agentes etiológicos como lesiones vasculares o neoplasias que puedan comprimir el recorrido nervioso, lo que facilita también un posterior tratamiento (De Laat, 2020). El objetivo del tratamiento inicial es el control del dolor, con medicamentos de primera línea como la carbamacepina, lamotrigina, amitriptilina, fenitoína, gabapentina y pregabalina (Di Stefano et al., 2018), como segunda elección se plantean intervenciones percutáneas, infiltraciones con anestésicos y los bloqueos al ganglio trigeminal (de Gasser), por último siendo más invasivo se encuentran la descompresión microvascular o rizotomia que en muchos casos han demostrado efectividad, (Liao et al., 2016; Lu et al., 2019) aunque las revisiones sistemáticas aun no reportan un tratamiento $100 \%$ efectivo en el control del dolor a largo plazo. (Zakrzewska et al., 2018). Teniendo en cuenta la importancia de estos síndromes dolorosos se propuso un estudio que describa la experiencia en el manejo de las neuralgias faciales en el Hospital Universitario San Ignacio, en Bogotá, Colombia.

\section{MATERIAL Y MÉTODO}

Previo aval del comité de Investigación y Ética de la Facultad de Odontología y Hospital Universitario San Ignacio (HUSI), se realizó un estudio descriptivo de las historias clínicas registrados en el sistema SAHI con los códigos CIE10, G50.0 Neuralgia Trigémino, G53.0 Neuralgia Post Herpética y G52.1 otros trastornos de neuralgia analizando sexo, edad, diagnóstico, exámenes complementarios, tratamiento(s) e información disponible de evolución. Los datos recolectados fueron registrados en una de hoja de cálculo de Excel y se analizaron mediante estadística descriptiva.

\section{RESULTADOS}

Se incluyeron 119 historias clínicas en el estudio, en la cuales se registró un $84 \%$ de pacientes con neuralgia de trigémino, $11,8 \%$ con neuralgia postherpética, 0,8 \% con neuralgia postherpética y trigéminal, 2,5\% con neuralgia glosofaríngeo y 0,8 \% con neuralgia glosofaríngeo y trigéminal. La distribución por sexo evidencia que el 72,3 \% fueron mujeres con 58 años en promedio con rango de 22 a 90 años) y el $27,7 \%$ fueron hombres con 65 años en promedio, con rango de 36 a 88 años). De acuerdo al sexo la neuralgía del trigémino fue más frecuente en mujeres, seguida de la neuralgia postherpética. (Fig. 1)

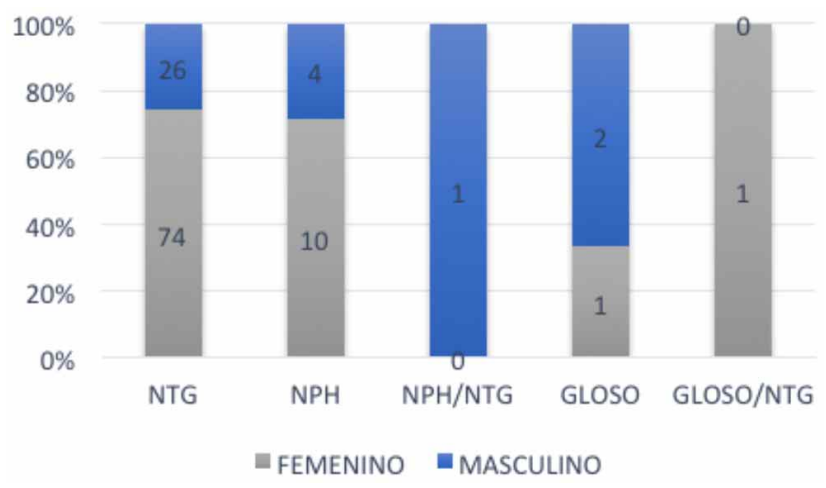

Fig. 1. Distribución de pacientes según sexo y neuralgias. NTG: Neuralgia trigémino; NPH: Neuralgia Postherpética; NPH/NTG: Neuralgia Trigémino/Neuralgia Postherpética; GLOSO: Neuralgia Glosofaríngeo; GLOSO/NTG: Neuralgia Glosofaríngeo / Neuralgia Trigémino.

En cuanto al lado afectado existe una tendencia a que el lado izquierdo sea el más frecuente ya que en la neuralgia trigéminal el $54 \%$ de estas se encontraban localizadas en este lado y tiende a afectar más a V2 y V3, mientras que en la neuralgia asociada al herpes zoster en el $64 \%$ de los pacientes se afecta el lado izquierdo con tendencia a ser mas de V1, mientras que la bilateralidad es solo del $3 \%$.

El $63 \%$ de las historias reportaban antecedentes médicos, siendo la hipertensión arterial la más frecuente en el 37,8 \%, depresión en el 8,4\%, hipotiroidismo en el 6,7 \% y diabetes en el 5,8 \%, lo que probablemente se asocie con la mayor edad de grupo estudiado.

En cuanto a las ayudas diagnósticas al 56,3 \% (67 pacientes) se les solicitó alguna ayuda, siendo más frecuente la resonancia magnética 38,8 \% (26 pacientes). De estos pacientes, 10 presentaron alguna malformación vascular descrita como ectasias, lo cual se podría asociar con HTA ya que de éstos 7 pacientes presentaban este antecedente, 1 presentó reporte de neuralgia del glosofaríngeo/neuralgia trigémino con diagnóstico de silla turca vacía, las restantes resonancias mostraban cambios anatómicos, quistes y lesiones benignas

Respecto al tratamiento farmacológico se observó el uso de varios tipos de medicamentos sólos o en combinación en común que incluían la gabapentina, 
LEÓN-CORREDOR, M.; FONSECA-RUBIO, M. A.; VELOSA-PORRAS, J.; RODRÍGUEZ-CIODARO, A. \& BARRIENTOS-SÁNCHEZ, S. Neuralgia del trigémino, del glosofaríngeo y postherpética en pacientes del Hospital Universitario San Ignacio (HUSI) Bogota, Colombia. Int. J. Odontostomat., 15(3):688-693, 2021.

fenitoina, oxcarbamazepina, amitriptilina, pregabalina, carbamazepina, imipramina, capsaicina, aciclovir sólo en neuralgia postherpética, aines y opioides, siendo los más frecuentemente utilizados en un 55,4 \% la carbamazepina, pregabalina y gabapentina un 26,9 \%. Las combinaciones más frecuentes en cada neuralgia de medicamentosse describen en la Tabla I.

El 33,6 \% del total de los pacientes se sometieron a tratamiento quirúrgico en la neuralgia de trigémino, 36 casos requirieron algún manejo quirúrgico como la radiofrecuencia (13 casos), rizotomía (6 casos), neurólisis (5 casos), descompresión micro vascular del nervio (2 casos), resección de lesión quística ( 2 casos), infiltración con lidocaína en ramas terminarles (1 caso), bloqueo de ganglio trigeminal (1 caso), también realizaron combinación de éstos como, rizotomía + resección quística (1 caso), descompresión microvascular + resección quística (1 caso), bloqueó de ganglio trigeminal + infiltración con lidocaína (1 caso), radiofrecuencia + infiltración de lidocaína (1 caso) radiofrecuencia + bloqueo del ganglio trigeminal (1 caso), bloqueo del ganglio trigeminal + resección quística (1 caso) y descompresión + radiofrecuencia (1 caso). En la neuralgia postherpética se aplicó toxina botulínica (1 caso) y bloqueo del nervio + parches lidocaína (1 caso), por último, en la neuralgia del glosofaríngeo la técnica quirúrgica utilizada fue infiltración con lidocaína ( 2 casos) y neurólisis (1 caso). Fig. 2.

El tiempo de seguimiento promedio en la neuralgia del trigémino fue de 3 años, en la neuralgia

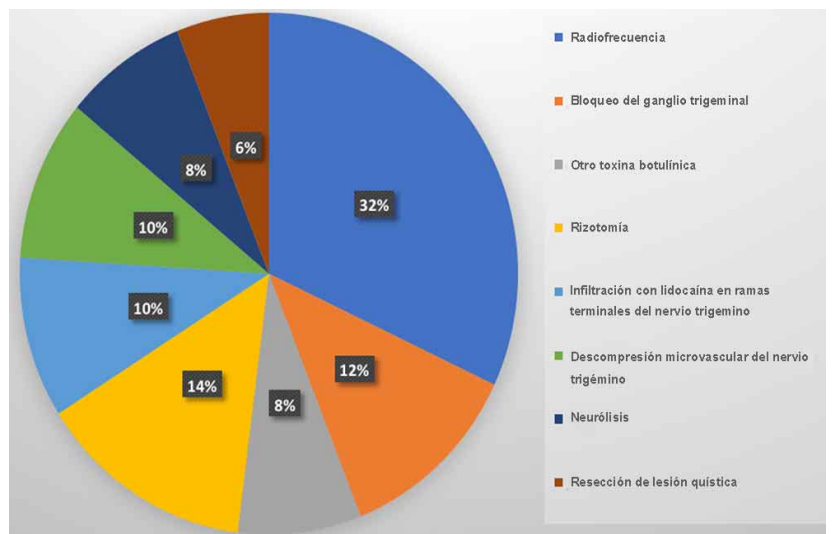

Fig. 2. Frecuencia y tipo de tratamiento quirúrgico en pacientes con diagnóstico de neuralgias.

postherpética un promedio de 2 años y de glosofaríngeo 1,5 años. En este tiempo de seguimiento, se observó en $25 \%$ de los casos reincidencia, de las cuales 29 correspondía a casos de neuralgia de trigémino, presentando 61 recaídas que iban desde 1 hasta 6 recaídas por paciente; 17 recaídas se presentaron en pacientes a los que previamente se les había realizado algún tratamiento quirúrgico. El tratamiento que se instauró para estas recidivas fue el aumento de la dosis en el medicamento en un $22,7 \%$, seguido de rizotomía con un 6,7\%, radiofrecuencia 5,9\% y bloqueo del ganglio de Gasser 5\%. Respecto a la neuralgia del glosofaríngeo sólo se registró una recaída tratada con la adición de un fármaco de mecanismo de acción diferente y el aumento de la dosis del medicamento base.

Tabla I. Frecuencia en la combinación de medicamentos de acuerdo con el tipo de neuralgia.

\begin{tabular}{|c|c|c|c|c|c|c|c|c|}
\hline Neuralgia & 1 Medicamentos & 2 Medicamentos & & 3 Medicamentos & 4 Medicamentos & 5 Medicamentos & & 6 Medicamentos \\
\hline NTG & $\begin{array}{l}\text { - Carbamazepina } \\
\text { - Pregabalina } \\
\text { - Gabapentina }\end{array}$ & $\begin{array}{l}\text { - Amitriptilina+ } \\
\text { Carbamazepina } \\
\text { - Pregabalina } \\
\text { Carbamazepina } \\
\text { - Gabapentina+ } \\
\text { Carbamazepina }\end{array}$ & + & $\begin{array}{l}\text { - Amitriptilina+ } \\
\text { Carbamazepina + } \\
\text { Gabapentina }\end{array}$ & $\begin{array}{l}\text { - Amitriptilina+ } \\
\text { Carbamazepina + } \\
\text { Aine+ Opioide. }\end{array}$ & $\begin{array}{l}\text { - Carbamazepina } \\
\text { + Pregabalina } \\
\text { Fenitoina } \\
\text { Gabapentina } \\
\text { Opioide }\end{array}$ & $\begin{array}{l}+ \\
+ \\
+\end{array}$ & $\begin{array}{l}\text { Gabapentina } \\
\text { Oxicodona/ } \\
\text { Amitriptilina } \\
\text { pregabalina+ } \\
\text { Carbamazepina+ } \\
\text { Aine }\end{array}+$ \\
\hline $\mathrm{NPH}$ & $\begin{array}{ll}\text { - } & n=39 \\
\text { - } & \text { Aciclovir } \\
\text { - } & \text { Carbamazepina }\end{array}$ & $\begin{array}{l}\mathrm{n}=41 \\
\text { - } \text { Aciclovir+ } \\
\text { Carbamazepina } \\
\text { - Carbamazepina } \\
\text { Gabapentina }\end{array}$ & + & $\begin{array}{l}\mathrm{n}=11 \\
\text { - } \text { Aciclovir }+ \\
\text { Carbamazepina }+ \\
\text { Amitriptilina }\end{array}$ & $\begin{array}{ll}\mathrm{n}=5 & \\
- & \text { Aciclovir }+ \\
& \text { Carbamazepina + } \\
& \text { Pregabalina }+ \\
& \text { Gabapentina }\end{array}$ & $n=1$ & & $n=2$ \\
\hline & $-n=5$ & $n=6$ & & $n=3$ & $n=1$ & & & \\
\hline NPH/NTG & $\begin{array}{l}\text { - Gabapentina } \\
n=1\end{array}$ & & & & & & & \\
\hline GLOSO & $\begin{array}{l}\text { - Carbamazepina } \\
\text { - } n=1\end{array}$ & $\begin{array}{l}\text { - Carbamazepina } \\
\text { Gabapentina } \\
n=6\end{array}$ & + & & & & & \\
\hline GLOSO/NTG & $\begin{array}{l}\text { - Carbamazepina } \\
\text { - } n=1\end{array}$ & & & & & & & \\
\hline
\end{tabular}


LEÓN-CORREDOR, M.; FONSECA-RUBIO, M. A.; VELOSA-PORRAS, J.; RODRÍGUEZ-CIODARO, A. \& BARRIENTOS-SÁNCHEZ, S. Neuralgia del trigémino, del glosofaríngeo y postherpética en pacientes del Hospital Universitario San Ignacio (HUSI) Bogota, Colombia. Int. J. Odontostomat., 15(3):688-693, 2021.

\section{DISCUSIÓN}

Los dolores neuropáticos, crónicos y severos impactan la calidad de vida, con una incidencia global de neuralgia de trigémino de $27 / 100.000$ / año (Burmeister et al., 2015), una incidencia para NPH de 3,4 personas por 1000 en los Estados Unidos (Liao et al.) y por último, la neuralgia del glosofaríngeo presentando por cada 100 casos de NT, 1 caso de NGF (Ibrahim et al., 2014), son importantes en el diagnóstico en odontología, porque requieren un cuidadoso análisis de antecedentes y examen clínico para derivar al paciente al especialista adecuado. Los resultados se asemejan a lo reportado en países asiáticos y de Estados Unidos, por cuanto se encontró una mayor prevalencia en mujeres siendo la neruralgia trigéminal la más frecuente, seguido de la neuralgia post herpética y neuralgia del glosofaríngeo (Jainkittivong et al., 2012; Singh et al.). Se encontraron dos casos de combinación entre neuralgias, uno correspondió a NT combinada con NGF y el segundo caso de NT con NPH, lo que no se ha reportado en otros estudios.

En esta muestra el lado izquierdo fue el más afectado tanto en la NT como en la NPH, lo que es inconsistente con la teoría de variación anatómica nombrada por quienes sugieren que en la mayoría de los casos de neuralgias del nervio trigémino se deben a que el foramen oval derecho del cráneo humano es más estrecho en relación al contralateral, causando una compresión de las ramas que emergen por él, y finalmente causar la neuralgia con alta tasa de incidencia en el lado derecho (Santo et al., 2005).

En cuanto a los antecedentes médicos concomitantes fue la HTA la patología más frecuente para los tres tipos de neuralgias, en segundo lugar los desórdenes psicológicos y con menos frecuencia la esclerosis múltiple, lo que concuerda en cierta parte con autores que refieren a la hipertensión arterial como la enfermedad sistémica más prevalente en pacientes con la NT, esto relacionado a que los desórdenes cardiovasculares pueden causar una vasodilatación en las arterias y venas, lo que desencadenaría una respuesta dolorosa al comprimir una parte del recorrido nervioso, desencadenando un contacto patológico entre el nervio y vaso. Por esto autores como Jainkittivong et al., afirman que la esclerosis múltiple y la hipertensión pueden ser vistos como únicos factores de riesgo claramente identificados con la neuralgia de trigémino (Jainkittivong et al.), pero otros sustentan que la hipertensión arterial en pacientes con neuralgia de trigémino no está directamen- te relacionada, si no que este antecedente médico puede ser una condición que coexiste en el paciente ya que tanto la hipertensión como la neuralgia son comunes en personas mayores. (Teruel et al., 2009). Los resultados en esta muestra evidencian una incidencia del $8 \%$ de pacientes con desórdenes psicológicos, lo que no es reportado en estudios previos en relación con la NT, en cuanto a la NPH la literatura sólo menciona la relacionan con pacientes inmunocomprometidos.

La resonancia magnética realizada en la mayoría de los casos, en esta última se hallaron algunas patologías, como lesiones benignas en ángulo ponto cerebeloso, infartos o atrofia cerebral, tortuosidades de arterias, dilataciones de arterias, entre otras. Este examen debería ser instaurado en un protocolo de atención por el aporte clínico, en pacientes con posible diagnóstico de neuralgia, para obtener imágenes claras y específicas del recorrido de los nervios y vasos sanguíneos que los acompañan (Yuan et al., 2016)

En este estudio todos los pacientes fueron tratados inicialmente con terapia farmacológica, siendo la carbamacepina la primera opción, y la American Academy of Neurology (AAN) señala que este medicamento es efectivo en la resolución del dolor entre el 58 al $100 \%$, por esto la AAN en conjunto con la Federación Europea de Sociedades Neurológicas la recomiendan como opción inicial en el manejo de neuralgias junto con los demás anticonvulsivantes. Esta actúa inhibiendo los canales de sodio sobre aquellas neuronas que sufren descargas, así como también tiene efecto sobre el sistema nervioso central disminuyendo la trasmisión sináptica. Los efectos adversos de este medicamento incluyen mareo, náuseas, estreñimiento, fatiga, somnolencia, visión borrosa, hepatotoxicidad y la supresión de la medula ósea se encuentran entre los eventos adversos de mayor gravedad a tener en cuenta dado que se trata de terapias a largo plazo. Por otro lado, la pregabalina, gabapentina y oxcarbazepina son usados como $2^{\mathrm{a}}$ y $3^{\mathrm{a}}$ elección según con lo referido en la literatura, siendo esta última la que presenta menos efectos adversos, con la misma eficacia que la carbamacepina por lo que para algunos autores es el medicamento de elección en el inicio del tratamiento (Green et al., 2019; Moore et al., 2019).

Opciones con el manejo de la toxina botulínica tipo $A$, en inyecciones subcutáneas, ha demostrado ser un tratamiento prometedor para el dolor en pacientes con pobre respuesta al tratamiento farmacológico convencional o una intervención 
LEÓN-CORREDOR, M.; FONSECA-RUBIO, M. A.; VELOSA-PORRAS, J.; RODRÍGUEZ-CIODARO, A. \& BARRIENTOS-SÁNCHEZ, S. Neuralgia del trigémino, del glosofaríngeo y postherpética en pacientes del Hospital Universitario San Ignacio (HUSI) Bogota, Colombia. Int. J. Odontostomat., 15(3):688-693, 2021.

neuroquirúrgica ineficiente.Sus efectos se describen con una duración de 3 meses aproximadamente, podría ser un tratamiento potencial beneficiando la calidad de vida de los pacientes con cualquiera de los tipos de neuralgias (Morra et al., 2016) en este estudio este tipo de tratamiento se usó en un sólo caso de NPH combinándola con gabapentina con un seguimiento a 3 años, mostrando efectos positivos.

En los casos en que los pacientes ingresaban por el servicio de urgencias presentando reincidencia, comúnmente eran tratados con opioides y aines, incluso en algunos casos, estos medicamentos se instauraban como tratamiento crónico, junto con el aumento en las dosis de los anticonvulsivantes. Esta polimedicación resultó, para la mayoría de ellos, en la mejoría del dolor neuropático pero la calidad de vida se vio afectada de otra forma ya que se presentaban efectos secundarios característicos a esta clase de medicamentos. En pacientes con recaída o hallazgos en la resonancia (tortuosidades arteriales, lesiones tumorales, infartos, entre otros) que puedan causar la neuralgia, el tratamiento concomitante más usado suele ser la radiofrecuencia, teniendo como objetivo lesionar las fibras nerviosas tipo $\mathrm{A}$ y $\mathrm{C}$ encargadas de la transmisión del dolor (Díaz et al., 2013). Esta técnica es bastante usada por los servicios de Neurocirugía y Neurología, combinándola también con radiofrecuencia por termocoagulación mostrando efectos positivos. La rizotomia y el bloqueo del ganglio trigeminal fueron la segunda elección quirúrgica, mostrando mejores resultados a largo plazo la primera, ya que se realiza la sección de la raíz nerviosa periférica comprometida llevando consigo la anestesia permanente. En el bloqueo del ganglio trigeminal se utilizaban sustancias como alcohol y glicerol, lo que en un tiempo indefinido mostraron recurrencias. Otro tratamiento realizado fue la infiltración con lidocaína en un porcentaje bajo,en las terminaciones nerviosas y en referencia a esto, se ha encontrado en la literatura, el uso de lidocaína mezclada con esteroides como la metilprednisolona más calcitonina, que prolongan el efecto en un promedio de 28 semanas (Elshiek et al., 2016).

Ante el hallazgo de patologías como lesiones neoplásicas se usó la resección de la lesión seguida de la descompresión microvascular, separando la arteria del nervio mediante materiales sintéticos como el teflón, estos son reportados como tratamientos eficaces en algunos casos, pero no siempre se obtienen resultados positivos a largo plazo porque este tipo de tratamientos invasivos lleva consigo efectos secundarios como parestesias, disestesias y anestesia de las ramas nerviosas afectadas, reportadas en los pacientes en este estudio.

Se concluye que las neuralgias faciales siguen siendo patologías de difícil manejo ya que aún en revisiones sistemáticas sobre su manejo, hay poca evidencia de la efectividad absoluta de alguno de los métodos empleados para su tratamiento y ninguno de ellos por separado evidencia ser mejor. Por eso, a menos que exista una patología asociada que deba ser tratada quirúrgicamente, la terapia farmacológica con los medicamentos aquí descritos, y usados desde los años 70, seguirá siendo la primera forma de su manejo dado su perfil de seguridad.

AGRADECIMIENTOS. Al Hospital Universitario San Ignacio por su invaluable colaboración de siempre.

LEÓN-CORREDOR, M.; FONSECA-RUBIO, M. A.; VELOSAPORRAS, J.; RODRÍGUEZ-CIODARO, A. \& BARRIENTOSSÁNCHEZ, S. Trigeminal, glossopharyngeal and post-herpetic neuralgia in patients of the San Ignacio University Hospital (HUSI) Bogota, Colombia. Int. J. Odontostomat., 15(3):688693, 2021.

ABSTRACT: To determine the clinical characteristics, guidelines for the diagnosis, treatment and evolution of trigeminal neuralgia, postherpetic neuralgia and glossopharyngeal neuralgia. A descriptive study of 119 clinical histories of patients diagnosed with Trigeminal Neoplasia, glossopharyngeal and postherpetic treated at the Hospital Universitario San Ignacio, recording data such as: gender, age, medical history, diagnostic exams, clinical features, branches and side of the face affected, treatments and evolution. 86 clinical records of women and 33 of men were evaluated, 100 with trigeminal neuralgia, 14 postherpetic, 3 glossopharyngeal, the most common affected side was the left side with $51 \%$, the most affected branches in trigeminal neuralgia was the $\mathrm{V} 2-\mathrm{V} 3$, postherpetic $\mathrm{V} 1$, the most frequent antecedent of the neuralgias was the AHT, the type of pain that was more frequent was the lancinanting with $82.4 \%$. The most commonly used medication was carbamazepine $(60.5 \%)$ and pregabalin $(29.4 \%)$; in surgical treatments radiofrequency was the most used in $32 \% ; 30$ patients had relapses that were generally managed with increased doses. This study shows a similarity with the reported literature, presenting more cases of trigeminal neuralgia, followed by the herpetic and finally the glossopharyngeal neuralgia, finding more cases in women than in men, with an average age of 60 years, affecting in Greater proportion the mandibular and maxillary branch in the trigeminal neuralgia and in the postherpetic ophthalmic.

KEY WORDS: trigeminal neuralgia, post-herpetic neuralgia, glossopharyngeal neuralgia, treatment of neuralgia. 


\section{REFERENCIAS BIBLIOGRÁFICAS}

Burmeister, J.; Holle, D.; Bock E.; Ose C.; Diener H. C. \& Obermann, $M$. Botulinum neurotoxin type $A$ in the treatment of classical Trigeminal Neuralgia (BoTN): study protocol for a randomized controlled trial. Trials, 16:550, 2015.

Cruccu, G.; Finnerup, N. B.; Jensen, T. S.; Scholz, J.; Sindou, M.; Svensson, P.; Treede, R. D.; Zakrzewska, J. M. \& Nurmikko T. Trigeminal neuralgia: New classification and diagnostic grading for practice and research. Neurology, 87(2):220-8, 2016.

De Laat, A. Differential diagnosis of toothache to prevent erroneous and unnecessary dental treatment. J. Oral Rehabil., 47(6):77581, 2020.

Díaz, R. C. R.; Benavides, J. L. B. \& Álvarez, J. G. Radiofrecuencia térmica del ganglio de Gasser en pacientes con neuralgia del trigémino. Rev. Col. Anest., 41(2):127-31, 2013.

Di Stefano, G.; Truini, A. \& Cruccu, G. Current and innovative pharmacological options to treat typical and atypical trigeminal neuralgia. Drugs, 78(14):1433-42, 2018.

Elsheikh, N. A. \& Amr, Y. M. Calcitonin as an additive to local anesthetic and steroid injection using a modified coronoid approach in trigeminal neuralgia. Pain physician, 19(7):457-64, 2016.

Green, T. H. \& Girgis F. Trigeminal neuralgia: Medical management and surgical options. J. Pain Palliat. Care Pharmacother., 33(12):32-3, 2019.

Ibrahim S. Trigeminal neuralgia: diagnostic criteria, clinical aspects and treatment outcomes. A retrospective study. Gerodontology, 31(2):89-94, 2014.

Jainkittivong, A.; Aneksuk, V. \& Langlais, R. P. Trigeminal neuralgia: a retrospective study of 188 Thai cases. Gerodontology, 29(2):e611-7, 2012.

Kawai, K.; Rampakakis, E.; Tsai, T. F.; Cheong, H. J.; Dhitavat, J.; Covarrubias, A. O.; Yang, L.; Cashat-Cruz, M.; Monsanto, H.; Johnson, K.; Sampalis, J. S. \& Acosta, C. J. Predictors of postherpetic neuralgia in patients with herpes zoster: a pooled analysis of prospective cohort studies from North and Latin America and Asia. Int. J. Infect. Dis., 34:126-31, 2015.

Liao, C.; Visocchi, M.; Yang, M.; Liu, P.; Li, S. \& Zhang, W. Pulsed radiofrequency: A management option for recurrent trigeminal neuralgia following radiofrequency thermocoagulation. World Neurosurg., 97:760.e5-760.e7, 2017.

Lu, W.; Wang, H.; Yan, Z.; Wang Y. \& Che H. Microvascular decompression for the treatment of neurogenic hypertension with trigeminal neuralgia. BMC Neurol., 19:341, 2019.

Moore, D.; Chong, M. S.; Shetty, A.; Zakrzewska, J. M. A systematic review of rescue analgesic strategies in acute exacerbations of primary trigeminal neuralgia. Br. J. Anaesth., 123(2):e385-e396, 2019.

Morra, M. E.; Elgebaly, A.; Elmaraezy, A.; Khalil, A. M.; Altibi, A. M.; Vu, T. L.; Mostafa, M. R.; Huy, N. T. \& Hirayama, K. Therapeutic efficacy and safety of botulinum toxin a therapy in trigeminal neuralgia: a systematic review and meta-analysis of randomized controlled trials. J. Headache Pain, 17(1):63, 2016.

Neto, H. S.; Camilli, J. A. \& Marques, M. J. Trigeminal neuralgia is caused by maxillary and mandibular nerve entrapment: greater incidence of right-sided facial symptoms is due to the foramen rotundum and foramen ovale being narrower on the right side of the cranium. Med. Hypotheses, 65(6):1179-82, 2005.

Sathasivam, H. P.; Ismail, S.; Ahmad, A. R.; Basri, N. N.; Muhamad, H.; Mohd Tahir, N. F.; Saw, C. L.; Hj Kipli, N. \& Lau, S. H. Trigeminal neuralgia: a retrospective multicentre study of 320 Asian patients. Oral Surg. Oral Med. Oral Pathol. Oral Radiol., 123(1):51-57, 2017.
Singh, P. M.; Kaur, M. \& Trikha, A. An uncommonly common: Glossopharyngeal neuralgia. Ann. Indian Acad. Neurol., 16(1):18, 2013.

Teixeira, M. J.; de Siqueira, S. R. \& Bor-Seng-Shu, E. Glossopharyngeal neuralgia: neurosurgical treatment and differential diagnosis. Acta Neurochir. (Wien), 150(5):471-475, 2008.

Teruel, A.; Ram, S.; Kumar, S. K. S.; Hariri, S. \& Clark GT. Prevalence of hypertension in patients with trigeminal neuralgia. J. Headache Pain, 10(3):199-201, 2009.

Yuan, Y.; Zhang, Y.; Luo, Q. I. \& Yu J. Trigeminal neuralgia caused by brain arteriovenous malformations: A case report and literature review. Exp. Ther. Med., 12(1):69-80, 2016.

Zakrzewska, J. M.; Wu, J. \& Brathwaite, T. S. A Systematic review of the management of trigeminal neuralgia in patients with multiple sclerosis. World Neurosurg., 111:291-306, 2018.

Dirección para correspondencia:

Silvia Barrientos Sánchez

Centro Investigaciones Odontológicas

Facultad de Odontología

Pontificia Universidad Javeriana

Bogotá

COLOMBIA

E-mail: barrien@javeriana.edu.co

León-Corredor Maryorie. orcid.org/0000-0003-3816-2294

Barrientos-Sánchez Silvia orcid.org/0000-0001-7280-3791

Rodríguez-Ciodaro Adriana orcid.org/0000-0002-6640-3975 